\title{
Propagation of acoustic waves in phononic-crystal plates and waveguides using a finite-difference time-domain method
}

\author{
Jia-Hong Sun and Tsung-Tsong Wu* \\ Ultrasonics Laboratory, Institute of Applied Mechanics, National Taiwan University, Taipei 106, Taiwan \\ (Received 12 February 2007; revised manuscript received 10 August 2007; published 17 September 2007)

\begin{abstract}
Propagation of acoustic waves in a phononic-crystal plate and related waveguides are analyzed in this paper. A two-dimensional phononic-crystal plate consisting of circular steel cylinders which form a square lattice in an epoxy matrix is studied first using the finite-difference time-domain (FDTD) method. The Bloch theorem is employed to deal with the periodic condition, and the traction free condition is set on the top and bottom boundaries of the plates. The dispersion curves and displacement fields are calculated to identify the band gaps and eigenmodes. With the existence of a complete band gap in the phononic-crystal plate, an acoustic waveguide is presented accordingly. Eigenmodes of acoustic waves inside the waveguides are indicated, and the modes are affected by the geometry arrangement of waveguides. Inside the phononic-crystal plate waveguides, wave propagation is well confined within the structure.
\end{abstract}

DOI: 10.1103/PhysRevB.76.104304

PACS number(s): 63.20.-e, 43.20.+g, 43.40.+s, 46.40.Cd

\section{INTRODUCTION}

Phononic crystals have attracted much academic interest in their property of band gaps during the past decade. A band gap is the frequency range that acoustic waves cannot propagate in a periodic structure. The bulk acoustic waves (BAWs) in the unbounded phononic crystals ${ }^{1-3}$ and the surface acoustic waves (SAWs) in the phononic crystals with a free surface $^{4-8}$ were analyzed. In these studies, band gaps of BAW and SAW were observed. Furthermore, complete band gaps that BAW and SAW are forbidden to propagate in any direction were also analyzed. With the properties of complete band gaps, many applications of phononic crystals were designed accordingly, such as elastic wave filters, couplers, ${ }^{9}$ and waveguides. ${ }^{9-13}$ A phononic-crystal waveguide is an important elementary component to build an acoustic wave circuit. In fact, to construct the SAW waveguides is more practical because the unbounded BAW waveguide is difficult to realize. However, the possible energy loss of pseudosurface acoustic wave (PSAW) in the SAW waveguides is another considerable issue. ${ }^{14,15}$

Recently, the acoustic wave propagation in the twodimensional phononic-crystal structure with a finite thickness referred to as a phononic-crystal plate was studied. ${ }^{16,17}$ The plate is constructed by periodic arranged elastic materials with two free surfaces. Unlike the PSAW in semi-infinite phononic-crystal media that allows energy leakage, the finite plate thickness keeps acoustic waves inside the structure. In this paper, we study the characteristics of acoustic wave propagating in the phononic-crystal plates and the related waveguides. The FDTD method is developed to analyze the dispersion of acoustic wave in the phononic-crystal plates. The Bloch theorem and traction free conditions are employed to deal with the periodic boundary condition and the top and bottom surfaces of plates, respectively. A steel/epoxy square lattice phononic-crystal plate is studied, and complete band gaps of acoustic wave are obtained. With the properties of the complete band gaps, a phononic waveguide is designed by arranging adjacent point defects. The eigenmodes of acoustic waves inside waveguides are indicated and the acoustic waves propagating inside the straight waveguide are demonstrated. The results show that the allowed surface waves can be propagated and well confined within the waveguide.

\section{ACOUSTIC WAVES IN A PHONONIC-CRYSTAL PLATE}

The FDTD method had been developed to analyze the BAW in infinite phononic crystals ${ }^{18,19}$ and SAW in semiinfinite structures. ${ }^{14,15}$ This method has the flexibility to construct a variety of two-dimensional (2D) and three-dimensional (3D) periodic structures, and the phenomena of acoustic waves propagating inside the structures can be investigated. A typical phononic-crystal plate is shown in Fig. 1(a). The cylindrical inclusions are arranged to form a square lattice on the $x_{1}-x_{2}$ plane. The lattice constant of the phononic crystal is denoted by $a$. In the $x_{3}$-axis direction, the thickness of the plate is defined by $h$, and the normal of the free surfaces of the plate is in the $x_{3}$ axis. To analyze acoustic waves in a phononic-crystal plate, the periodic boundary condition based on Bloch's theorem and the traction free surface condition for the free surfaces are applied in the FDTD method.

The FDTD method for acoustic waves is developed from the equations of motion and the constitutive law of elastic materials. They are expressed as

$$
\begin{gathered}
\rho \ddot{u}_{i}=\tau_{i j, j}+\rho f_{i}, \\
\tau_{i j}=C_{i j k l} \varepsilon_{k l},
\end{gathered}
$$

where $\rho$ is the density of materials, $u_{i}$ is the displacement, $\tau_{i j}$ is the stress, $f_{i}$ is the body force, $C_{i j k l}$ is the elastic constant, and $\varepsilon_{k l}$ is the strain. With the definition of staggered grids, differential equations (1) and (2) are transferred into difference equations based on Taylor's expansion, and then the 3D heterogeneous finite-difference formulation is developed. By arranging the densities and elastic constants, these equations are applicable to the inhomogeneous structure of phononic crystals. Bloch's theorem is then introduced to treat the periodic boundary condition of a unit cell of the phononic 


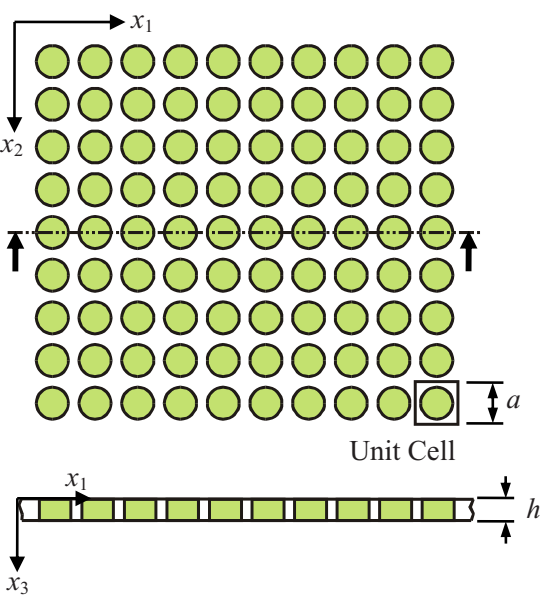

(a)

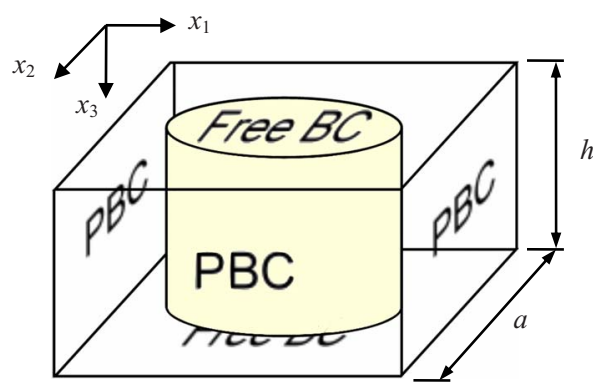

(b)

FIG. 1. (Color online) (a) The phononic-crystal plate with circular cylinders embedded in a background material arranged in a square lattice. (b) The unit cell of the phononic-crystal plate and setting of boundary conditions.

crystals. ${ }^{18}$ The direct periodic boundary conditions (PBCs) in terms of the displacement $u_{i}$ and the stress $\tau_{i j}$ are defined as ${ }^{19}$

$$
\begin{gathered}
u_{i}(\mathbf{x}+\mathbf{a}, t)=e^{i \mathbf{k} \cdot \mathbf{a}} u_{i}(\mathbf{x}, t), \\
\tau_{i j}(\mathbf{x}+\mathbf{a}, t)=e^{i \mathbf{k} \cdot \mathbf{a}} \tau_{i j}(\mathbf{x}, t),
\end{gathered}
$$

where $\mathbf{k}$ is a wave vector, and $\mathbf{a}$ is a lattice translation vector. Further, the traction free condition is defined on the top and bottom surfaces of the plates:

$$
\left.\tau_{3 j}(\mathbf{x}, t)\right|_{x_{3}=0}=\left.\tau_{3 j}(\mathbf{x}, t)\right|_{x_{3}=h}=0 .
$$

Figure 1(b) is the unit cell of the square lattice phononiccrystal plates, and the setting definitions of the boundaries are applied to the FDTD method. With the periodic boundary condition, the unbounded periodic structures can be analyzed by calculating a unit cell; furthermore, the property of phononic-crystal plates is available with the definition of traction free surfaces. In the unit cell, a small disturbance in a random position is set as the initial condition. Thus, all possible wave modes are excited inside the considered phononic-crystal plate. The time-varying displacement is recorded during the calculations, and the Fourier transforma- tion is then performed to obtain the frequency spectrum. The eigenfrequencies of a given wave vector $\mathbf{k}$ are indicated by selecting the resonance peaks of the spectrum.

In this paper, a square lattice phononic-crystal plate consisting of steel cylinders embedded in an epoxy matrix is analyzed, and then it is also used to construct the waveguides. The material constants used in calculation are $\rho=7900 \mathrm{~kg} / \mathrm{m}^{3}, \mathrm{C}_{11}=280.2 \mathrm{GPa}$, and $\mathrm{C}_{44}=82.9 \mathrm{GPa}$ for steel, and those for epoxy are $\rho=1180 \mathrm{~kg} / \mathrm{m}^{3}, \mathrm{C}_{11}$ $=7.61 \mathrm{GPa}$, and $\mathrm{C}_{44}=1.59 \mathrm{GPa}$. To calculate the eigenmodes of acoustic waves in the phononic-crystal plate, the unit cell in Fig. 1(b) is defined. The cross section on the $x_{1}-x_{2}$ plane is $1 a \times 1 a$, and $a$ is the lattice constant. $a$ is divided into 48 grids in this study. The lattice constant $a$ of the phononic crystal is defined as $8 \mathrm{~mm}$, and the radius $r$ of steel cylinder is $3 \mathrm{~mm}$. Thus, a time step interval of $10 \mathrm{~ns}$ in the FDTD calculation is sufficient to satisfy the numerical stability condition.

In this case, the filling fraction, defined as $\pi r^{2} / a^{2}$, equals to 0.442 . In general, the eigenmodes of acoustic waves in phononic crystals are decided by the filling fraction, while the materials and geometry conditions (i.e., lattice symmetry) are chosen. However, the values of the eigenfrequencies of acoustic waves in the phononic-crystal plates are not only a function of filling fraction but also the plate thickness. ${ }^{16,17}$ In this paper, we chose the thickness $h$ as $0.25 a$ for the following study of waveguides.

To investigate the band gap of the phononic-crystal plate, the dispersion relations of acoustic waves are calculated first. Although the phononic-crystal plate is actually a $3 \mathrm{D}$ problem, the wave propagation is mainly confined within the $x_{1}-x_{2}$ plane. Thus, the first Brillouin zone and the denotation of the smallest irreducible area in $\mathbf{k}$ space for $2 \mathrm{D}$ cases are inherited here; moreover, the denotations are denoted with a bar on top to present the surface Brillouin zone symmetry points. The dispersion is shown in Fig. 2 and inset is the first Brillouin zone. In Fig. 2, there are three developing bands starting from the zero frequency to higher frequency range which are similar to the results of BAW. ${ }^{15}$ In addition, two noticeable complete band gaps appear from 89 to $101 \mathrm{kHz}$ and 125 to $162 \mathrm{kHz}$, respectively.

In an unbounded 2D phononic crystal, the structure is infinite along the $x_{3}$ direction. Thus, the BAW propagating within the $x_{1}-x_{2}$ plane can be decoupled into the in-plane modes $\left(x_{1}-x_{2}\right.$ plane) and the antiplane modes. However, in the phononic-crystal plate, the eigenmodes cannot be classified in the same way. The modes in the phononic-crystal plates are coupled, and they are identified as flexural (antisymmetric), longitudinal (symmetric), and transverse (shear horizontal) waves such as Lamb waves in the classical plates.

To investigate the acoustic wave modes in the dispersion, the transmission of waves and the polarization of specific modes are analyzed. First, to launch a wide frequency wave packets along $\bar{\Gamma} \bar{X}$ direction, line sources of $x_{2}$ and $x_{3}$ polarizations are defined on the plate surface, respectively. The acoustic waves propagating through a ten-layered phononiccrystal plate and a homogeneous plate without steel cylinders are recorded and compared with each other to calculate the transmission. The corresponding results are plotted in the left 


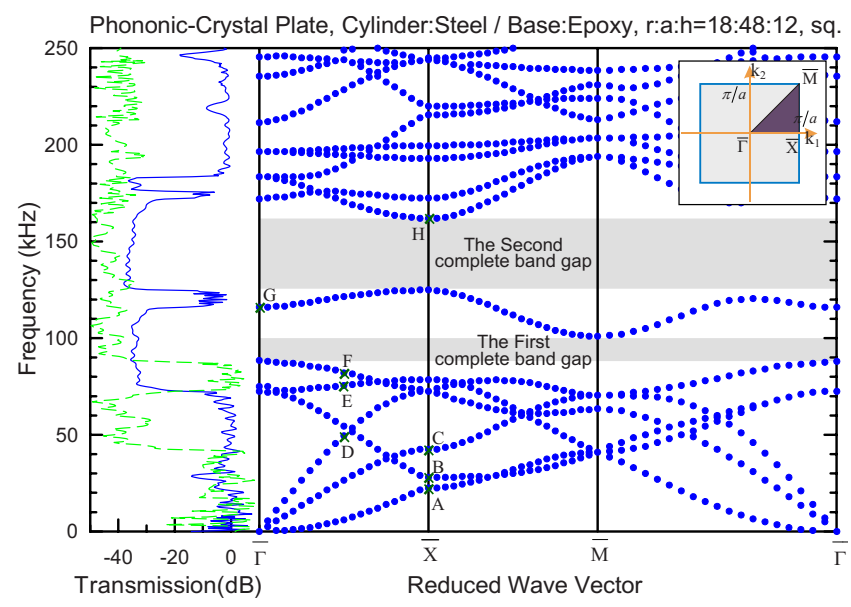

FIG. 2. (Color online) Band structure of the steel/epoxy phononic-crystal plate with square lattice. The filling fraction is 0.442 and plate thickness is $0.25 a$. The transmission of acoustic waves along $\bar{\Gamma} \bar{X}$ direction is also plotted on the left part. The solid and dotted lines present result of wave sources polarized along the $x_{3}$ and $x_{2}$ directions, respectively.

part of Fig. 2. When the wave is generated from the $x_{3}$-direction polarized source, the gaps appear at 72-116, $125-172$, and $176-183 \mathrm{kHz}$, shown by the solid line. The dotted line presents that, when the wave is generated from the $x_{2}$-polarized source, the passbands are between $73-87 \mathrm{kHz}$ and the range below $43 \mathrm{kHz}$. Compared to the dispersion of $\bar{\Gamma} \bar{X}$-direction, these two transmission distributions show a consistent result with the band structure, except a deaf band that can be observed on the top of the second complete band gap.

The displacement distribution inside the unit cell is calculated to investigate the eigenmodes of acoustic waves. The setup is the same as Fig. 1(a) but the initial condition is now replaced by a monochromatic wave source. The source generates a wave package with the chosen eigenfrequency, and the corresponding wave vector is set at the periodic boundary condition. By assigning both of the wave vector and the frequency, the specific eigenmode is, therefore, excited, and the displacement field is recorded to identify the modes.

The displacement distributions of the eigenmodes which belong to the first seven bands at $\bar{\Gamma} \bar{X}$ direction are plotted in Fig. 3 to investigate the wave propagating inside the plate. In these $3 \mathrm{D}$ plots, the directions of cones indicate the direction of displacement vector, and the sizes of the cones reflect the magnitudes of displacement vectors. First, the eigenmodes of wave vector $\mathbf{k}=(\pi / a, 0)$ with frequencies $f=22.5 \mathrm{kHz}$ (point $A$ in Fig. 2) and $f=28 \mathrm{kHz}$ (point $B$ in Fig. 2) are calculated, and the displacement distributions are shown in Figs. 3(a) and 3(b). These two modes belong to the lowest band and its folded band with the same wave vector. Since the wavelength of the first band with $|\mathbf{k}|=\pi / a$ is $2 a$, and thus, only one-half of the periodic displacement field is shown. In these two cases, the displacement fields are almost invariant along the $x_{2}$ axis. The 3D vector plots show the displacement distribution inside a unit cell. Additional 2D figures are also plotted for clearer understanding. The 2D figures show the polarizations along the plane which is parallel to $x_{1}-x_{3}$ plane and passing through the center of the steel cylinder. Obviously, the displacement components $u_{1}$ and $u_{3}$ dominate the behavior of these modes, which are basically corresponding to the lowest flexural mode in a plate.

The next eigenmode belongs to the second band in the phononic-crystal plate, and it has a wave vector $\mathbf{k}$ $=(\pi / a, 0)$ and $f=42.5 \mathrm{kHz}$ (i.e., point $C$ in Fig. 2). The displacement field on the plate at $x_{3}=h / 2$ is shown in Fig. 3(c). The eigenmode has a primary polarization in the $x_{2}$ direction. This is the lowest mode of horizontally polarized shear wave that the distribution does not vary obviously along thickness.

The case of the third band has a vector $\mathbf{k}=(\pi / 2 a, 0)$ and $f=49.5 \mathrm{kHz}$ (i.e., point D in Fig. 2). The mode has a higher phase velocity than the previous two bands. The distribution of the displacements is shown in Fig. 3(d), and the polarization on the plane $x_{3}=h / 2$ of the plate is also plotted. The polarization in this case is mainly along the $x_{1}$ direction, and the field does not have a large change along the thickness of the plate. This means it is the lowest longitudinal mode.

The modes of the next two bands are the waves of $\mathbf{k}$ $=(\pi / 2 a, 0)$ with $f=75.5 \mathrm{kHz}$ (point $E$ in Fig. 2) and $f$ $=82 \mathrm{kHz}$ (point $F$ in Fig. 2), respectively. For the mode of point $E$, the slice of typical displacement field is parallel to the $x_{2}-x_{3}$ plane and passes through the center of the steel cylinder, as shown in Fig. 3(e). The polarization remains at the $x_{2}-x_{3}$ plane but the magnitude also varies with the $x_{1}$ direction. Basically, this mode has $x_{3}$ components, but they are antisymmetric with respect to the $x_{1}-x_{3}$ plane passing the center of the unit cell. Thus, this band cannot be excited by the $x_{3}$-polarization line source, as shown in the transmission of Fig. 2. The result of point $F$ is shown in Fig. 3(f), and this band determines the lower boundary of the first complete band gap. The displacement distribution shows a circular polarization pattern on the plate at $x_{3}=h / 2$, and this band is excitable by the $x_{2}$-polarization source.

The next mode shown in Fig. $3(\mathrm{~g})$ is the mode with $\mathbf{k}$ $=(0,0)$ and $f=116 \mathrm{kHz}$ (point $G$ in Fig. 2) which exists in between the two complete band gaps. The normalized displacement fields of two slices $a-a^{\prime}$ and $b-b^{\prime}$ are plotted, respectively, in order to show the polarization clearly. Basically, the displacement field shows the property as a flexural mode in a plate, similar to the case shown in Fig. 3(b), but the field varies significantly along the $x_{2}$ direction.

Finally, an example of the deaf band observed in the transmission is calculated. The mode of $\mathbf{k}=(\pi / a, 0)$ and $f$ $=162 \mathrm{kHz}$ (point $H$ in Fig. 2) is shown in Fig. 3(h), and normalized displacement distributions of the unit cell and the two slices a-a' and b-b' are plotted. The displacement field is antisymmetric with respect to the $x_{1}-x_{3}$ plane passing through the central line of the unit cell. The $x_{3}$ component is antisymmetric and the $x_{2}$ component shows two opposite directions across the unit cell. Thus, neither the $x_{2}$ polarization nor the $x_{3}$ polarization line sources on the plate surface can excite this wave, and this band is identified as a deaf band.

Compared to the results of BAW reported in Ref. 15, the complete band gaps of BAW are at 90-143 and $153-204 \mathrm{kHz}$; the phononic-crystal plate has narrower complete band gaps than ones of BAW in the $2 \mathrm{D}$ case of the 

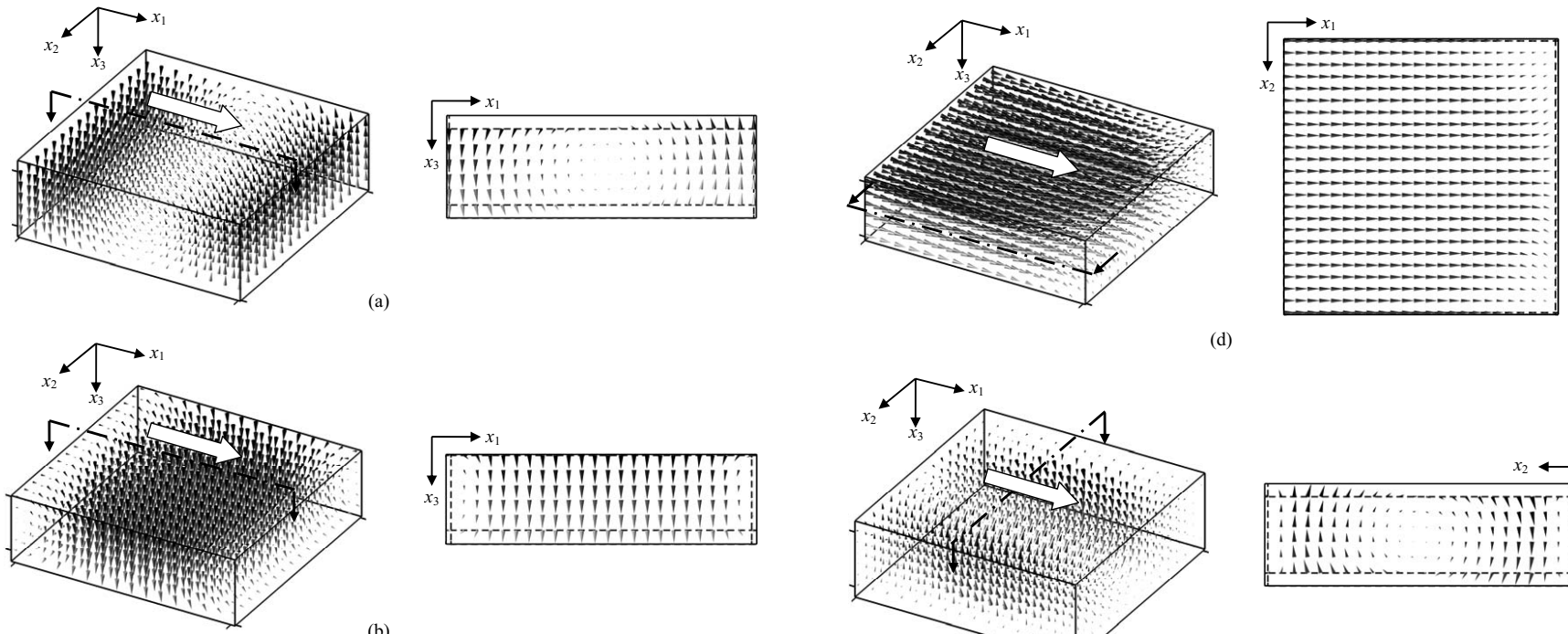

(b)

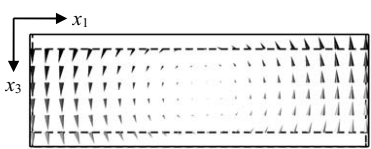

(a)

(d)
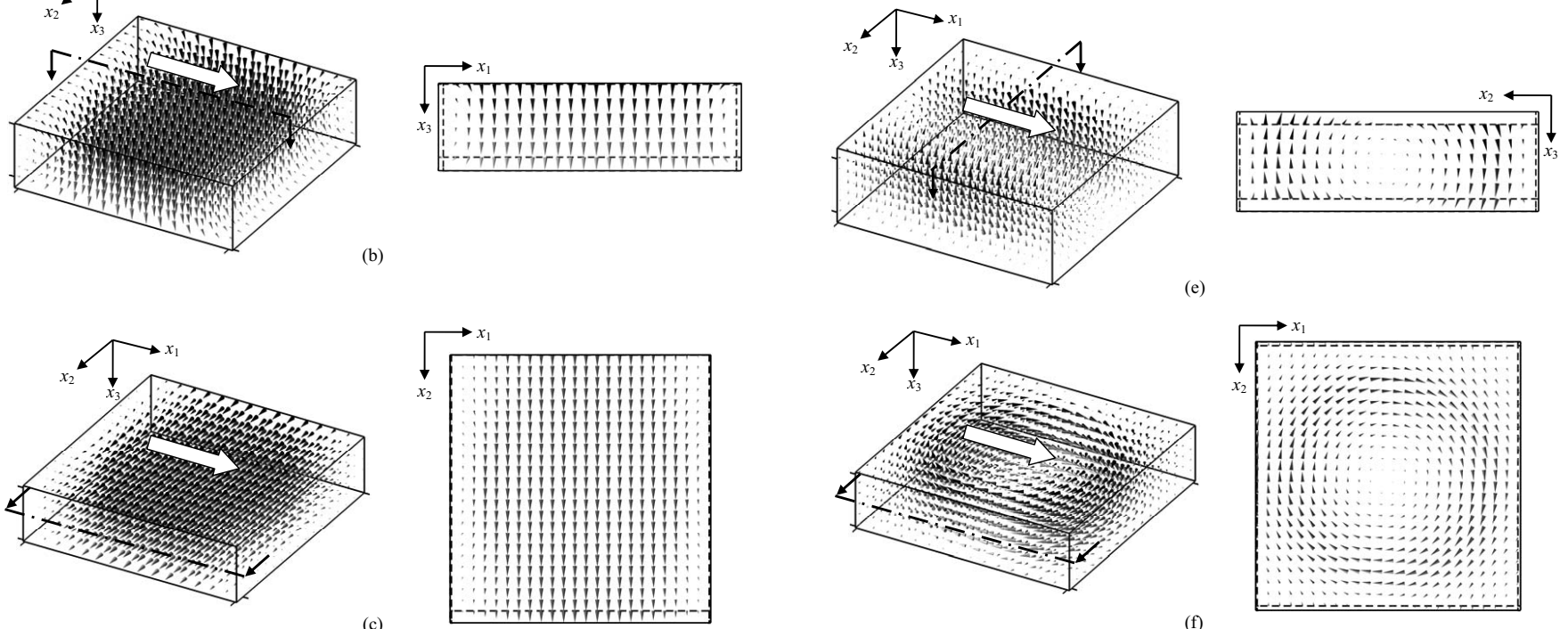

(c)
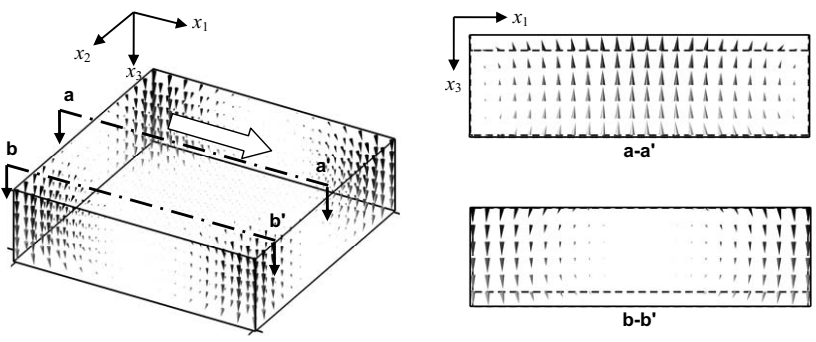

(g)
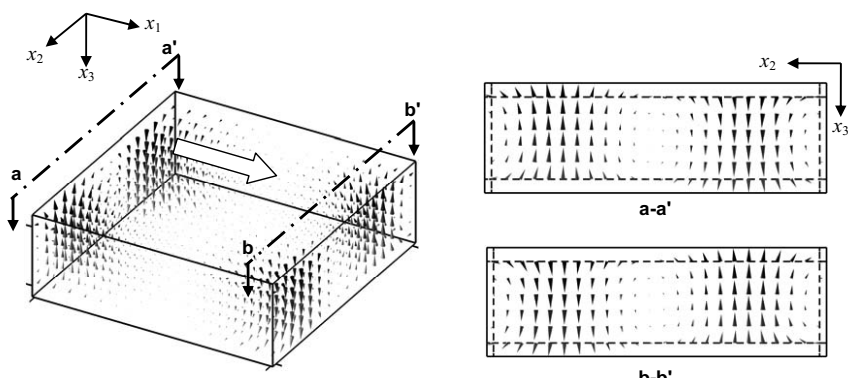

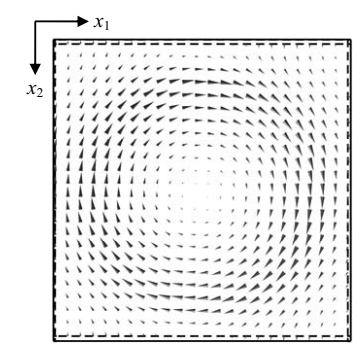

(f) 
ranges are 90-134 and 146-190 kHz. The calculation shows that in these phononic-crystal plates, the bands of longitudinal waves (the band of point $D$ in Fig. 2) and transverse waves (the bands of points $C$ and $F$ in Fig. 2) appear at similar ranges. Thus, the plate thickness does not alter the gaps of these two types of waves. However, the flexural modes (the bands of points $A, B, E, G$, and $H$ in Fig. 2) in the plates are affected by the thicknesses. In a thinner plate, the lowest flexural mode shifts toward the lower frequency range. Thus, the folded bands above the gaps of longitudinal and transverse waves shift downward and narrow the gap width. For a thicker plate, the cutoff frequency of higher order flexural modes is lowered, and then, the higher order modes appear at the range of the band gap. Therefore, the phononic-crystal plates usually have narrower complete band gaps than those of the 2D phononic crystal at the same filling fraction.

In brief, the dispersion curves, the transmissions, and the displacement distribution of the eigenmodes can be analyzed by the FDTD method. The displacement analyses help identify the polarizations of acoustic wave modes and verify the results of transmissions. In this phononic-crystal plate, the acoustic waves have not only similar modes as the Lamb modes in a classical plate but also some more complex modes because of the periodic cylinders. Furthermore, with a plate thickness as thin as $0.25 a$, the acoustic waves of the higher modes which vary along the thickness do not appear at the concerned frequency range. This simplifies the band structure and results in the obvious complete band gaps. Finally, the steel/epoxy phononic-crystal plate shows complete band gaps, and then it can be employed to create a waveguide in the next section.

\section{ACOUSTIC WAVES IN A PHONONIC-CRYSTAL PLATE WAVEGUIDE}

A waveguide is one of the important applications of phononic crystals for its unique role in acoustic circuits. To construct a waveguide, continuous point defects are defined in the phononic-crystal structure, as shown in Fig. 4(a). These defects form an area without scatters, i.e., the cylinders, and thus, the acoustic waves can propagate accordingly. Currently, most studies focus on the BAW in phononiccrystal waveguides, and few analyses of SAW in waveguides are reported. In this section, the acoustic waveguides in the phononic-crystal plates are analyzed with the FDTD method.

A phononic-crystal waveguide is formed based on the complete band gaps of acoustic waves. In the steel/epoxy phononic-crystal plate presented in Sec. II, there exist complete band gaps in 89-101 and $125-162 \mathrm{kHz}$. Thus, the plate can be used to create waveguides. Inside the waveguides, acoustic waves cannot penetrate across the boundaries. The existence of waveguides means that the defects are created in the perfect phononic-crystal structure, and thus the passbands are splitting off from its original value. At the bottom of a band gap, the gap-edge band splits into a higher frequency mode into the band gap. Similarly, the gap-edge band at the top of a band gap also splits into the band gap. Thus, these split modes contribute to defect modes (or guided

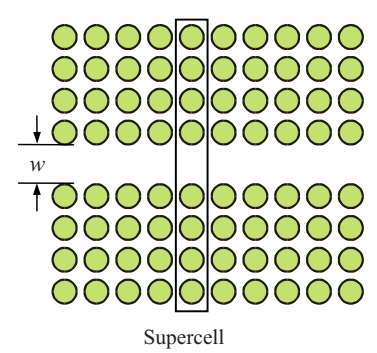

(a)

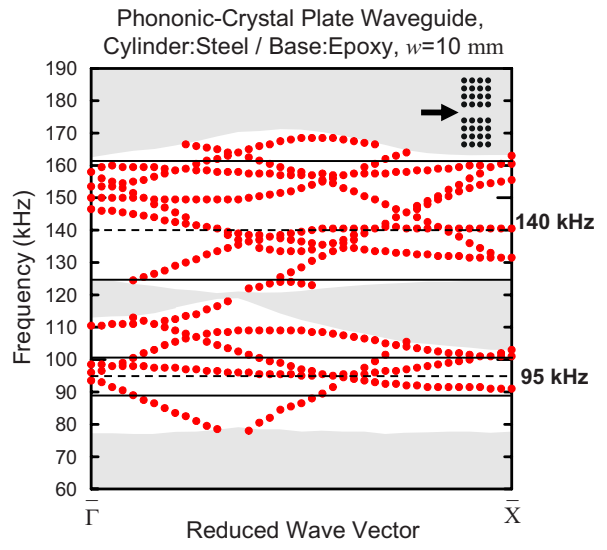

(b)

FIG. 4. (Color online) (a) Top view of a waveguide in the phononic-crystal plate and the supercell. (b) Band structure of acoustic waves in the steel/epoxy phononic-crystal plate waveguide of width $w=10 \mathrm{~mm}$.

modes) in the waveguides, and the defect modes will appear at the range of complete band gaps. ${ }^{20}$

In the FDTD method, the supercell technique is used to analyze the dispersion of a waveguide. A supercell is defined by combining several unit cells and then the whole supercell is treated as a basic cell in the analysis. As shown in Fig. 4(a), a supercell for the waveguides is chosen to contain the defect area and extra ten unit cells. Therefore, according to the definition of the supercell, there are ten rows of periodic phononic-crystal structure between two waveguides in our analysis. After defining the supercell and using the similar boundary conditions shown in Fig. 1(b), the wide band wave sources are then excited and the eigenmodes are analyzed by selecting the local maximum peaks from the Fourier transformed spectra as the procedure for a unit cell in Sec. II.

First, we consider a waveguide with a width $w=10 \mathrm{~mm}$, i.e., the distance between two neighboring cylinders on both sides of a waveguide. This means that one row of cylinders in the supercell is removed from the perfect periodic structure. Thus, a supercell of phononic-crystal plate consisting of 11 unit cells is defined to calculate eigenmodes of waveguide. The dispersion of the waveguide of $w=10 \mathrm{~mm}$ in the plate is obtained and shown in Fig. 4(b) with an inset on the top right to present the structure. This figure is focused on the frequency range of $60-190 \mathrm{kHz}$ to observe defect modes in the complete band gaps, and the boundaries of complete band gaps are marked with horizontal solid lines. In the waveguide, there are numerous extended modes at the pass- 
band of the phonic crystal, i.e., outside the range of complete band gaps. In this case, they appear at the range below $79 \mathrm{kHz}$, the range of $101-125 \mathrm{kHz}$, and the range above $162 \mathrm{kHz}$. These modes are not concerned and the regions are presented in gray.

In the band structure of acoustic waves in the phononiccrystal plate shown in Fig. 2, band gaps at $\bar{\Gamma} \bar{X}$ direction appear at 89-116 and $125-162 \mathrm{kHz}$. However, a passband locates at $101-125 \mathrm{kHz}$ along the $\bar{X} \bar{M}$ direction; therefore, the first complete band gap is actually in the range 89-101 kHz. In this case, acoustic waves propagating toward $\bar{\Gamma} \bar{X}$ direction have a larger partial band gap than the complete band gap. The additional partial band gap cannot result in useful guided waves because the acoustic waves at that frequency range will leave the waveguide along the $\bar{\Gamma} \bar{M}$ direction. In Fig. 4(b), the band structure of the waveguide is analyzed along the $\bar{\Gamma} \bar{X}$ direction, and the result shows that extended bands are allowed in the frequency range of $101-125 \mathrm{kHz}$, instead of the range of $116-125 \mathrm{kHz}$. This verifies the necessary of complete band gaps to form a waveguide. Besides, the upper limit of the lowest extended band in Fig. 4(b) is not $89 \mathrm{kHz}$, which is the highest value of sixth band at the $\bar{\Gamma} \bar{X}$ direction shown in Fig. 2, but the highest value $79 \mathrm{kHz}$ of the fifth band. The reason is that, as shown in Fig. 3(f), the acoustic waves of the sixth band polarized around the cylinder, and thus the mode does not appear in the waveguide. As a result, the useful frequency range for constructing the waveguides is still the same as the complete band gaps. Besides, some branches of the guided modes extend from the complete band gaps to the outside range. Acoustic waves that belong to these modes outside the complete band gaps can be excited. However, they convert into other transmission modes and propagate away from the waveguide when these modes encounter the waveguide boundaries. Thus, the modes outside the complete band gap still do not contribute to well propagation of waves in the waveguides.

In Fig. 4(b), there are 13 recognizable defect bands appearing in the complete band gaps. We did not examine all of the defect bands of phononic-crystal waveguides to indicate if they are active or deaf. In general, numerous defect bands at the same frequency range support the acoustic waves to propagate inside the waveguide. In the FDTD method, an area of $20 a \times 11 a$ is defined and the waveguide of $w$ $=10 \mathrm{~mm}$ is constructed to demonstrate the wave propagation. A monochromatic line source is set in the left-hand inlet of the waveguide and the acoustic waves propagate toward the right hand side. As illustrating cases, the waves generated from 95 and $140 \mathrm{kHz}$ sources with the $x_{3}$ polarization are calculated. These two frequencies belong to the first and the second complete band gaps, respectively. The result shows that wave propagation is confined within the scatter-free area. Therefore, these two complete band gaps are applicable to construct waveguides. These frequencies are marked with dashed lines in Fig. 4(b), and the figure shows that both the monochromatic wave sources of 95 and $140 \mathrm{kHz}$ can excite multiple defect modes inside the waveguide. Thus, the amplitude distribution shows a complicated pattern, not a simple harmonic wave.

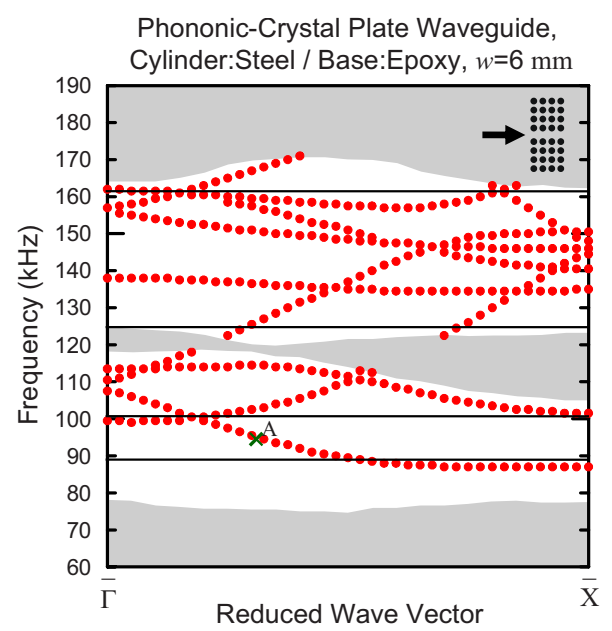

FIG. 5. (Color online) Band structure of acoustic waves in the steel/epoxy phononic-crystal plate waveguides of width $w=6 \mathrm{~mm}$.

However, a waveguide with a single mode or fewer defect modes is much preferred for some further applications. In the study of BAW in 2D phononic-crystal waveguide which has a narrower width allows fewer defect modes. ${ }^{9,11}$ In this study, we also depress the number of defect modes by reducing the width of the waveguide. The narrowed waveguides with a width $w=6 \mathrm{~mm}$ is analyzed, and the band structures are presented in Fig. 5. In the dispersion relations, the number of the bands at the range of complete band gaps is reduced from 13 to 10 . In the first complete band gap, $89-101 \mathrm{kHz}$, there are only two bands of defect modes. Similar to the abovementioned procedure, a $95 \mathrm{kHz}$ wave source is set in the inlet of waveguide to investigate the propagation of acoustic wave. In this case, the amplitude distribution on the surface at $x_{3}=0$ is shown in Fig. 6(a). In the plot, darker color means larger amplitude. The acoustic wave propagation inside the waveguide has a simple pattern. From the dispersion, the defect mode is marked as point $A$ in Fig. 5 and it belongs to a folded band. We found that the corresponding wave vector $\mathbf{k}$ is $(1.69 \pi / a, 0)$, and thus the wavelength is about $1.18 a$, which agrees with result of the displacement pattern. The characteristic displacement field is also plotted in Fig. 6(b). The 3D vector plot shows a full view of the waveguide, and the 2D figure of the cross section at the center of waveguide helps identify the wave as a flexural mode. This mode shows symmetric $x_{3}$ component with respect to the $x_{1}-x_{3}$ plane passing through the central line of the waveguide, and thus it is excitable by the line source of flexural waves.

The acoustic waves perform fewer bands in the waveguides with $6 \mathrm{~mm}$ width at the first complete band gap. However, there are still numerous defect modes at the second complete band gap of $125-162 \mathrm{kHz}$. Further, we calculate another narrowed waveguide of width $w=4 \mathrm{~mm}$. This means the waveguide results from an additional space of onequarter of a lattice constant between adjacent unit cells. The band structure is calculated and shown in Fig. 7. Because of the extremely narrowed width, there are only six bands inside the waveguide. Both two complete band gaps have simple band structures, and in addition, the range of 93$101 \mathrm{kHz}$ in the first complete band gap allows no defect 


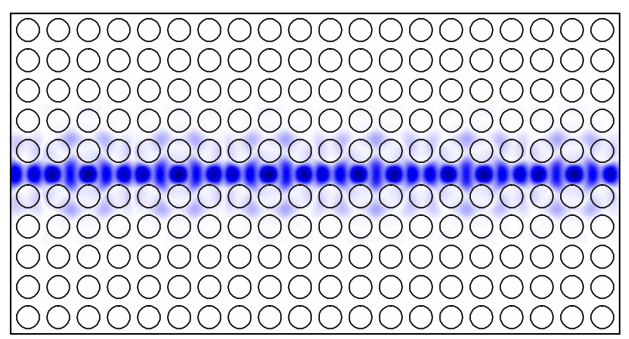

(a)
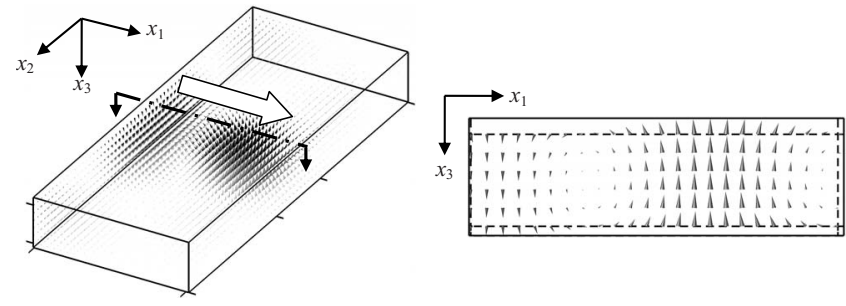

(b)

FIG. 6. (Color online) (a) Amplitude distribution of the $95 \mathrm{kHz}$ acoustic wave (point $A$ in Fig. 5) on the surface of the linear waveguide. (b) The displacement fields of the eigenmode point $A$ in Fig. 5.

modes. The consistent phenomenon is obtained because we observed that the $95 \mathrm{kHz}$ waves cannot propagate into the waveguide. Thus, more defect modes are depressed by constructing a narrower width waveguide, and the width of the waveguide cannot be too small in practice for the acoustic wave propagation.

Although the waveguides with a narrowed width have fewer modes, however, these waveguides are no longer consisted of the multiply unit cells. This is inconvenient to construct an acoustic circuit in a periodic phononic crystal, especially to create a bend waveguide. We proposed another modified waveguide by inserting scatters in the center of waveguides, as shown in Fig. 8(a). The waveguide has a width of $10 \mathrm{~mm}$ and the scatters in the center of the wave-

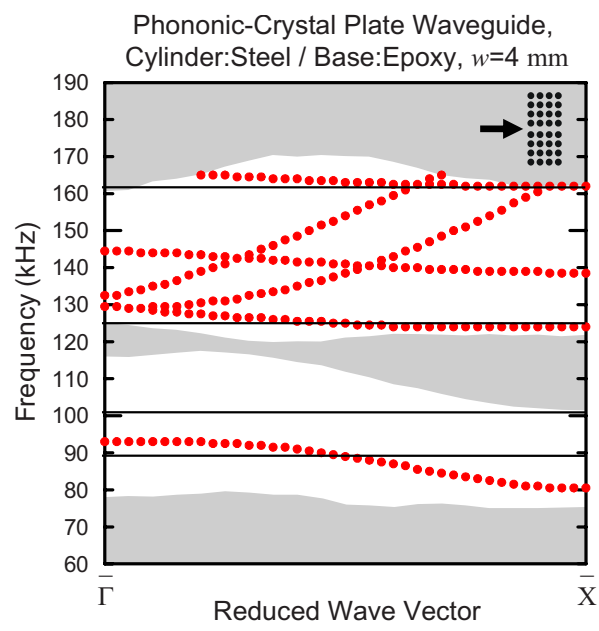

FIG. 7. (Color online) Band structure of acoustic waves in the steel/epoxy phononic-crystal plate waveguides of width $w=4 \mathrm{~mm}$.

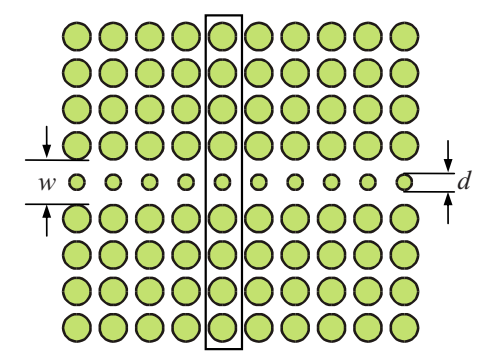

Supercell

(a)

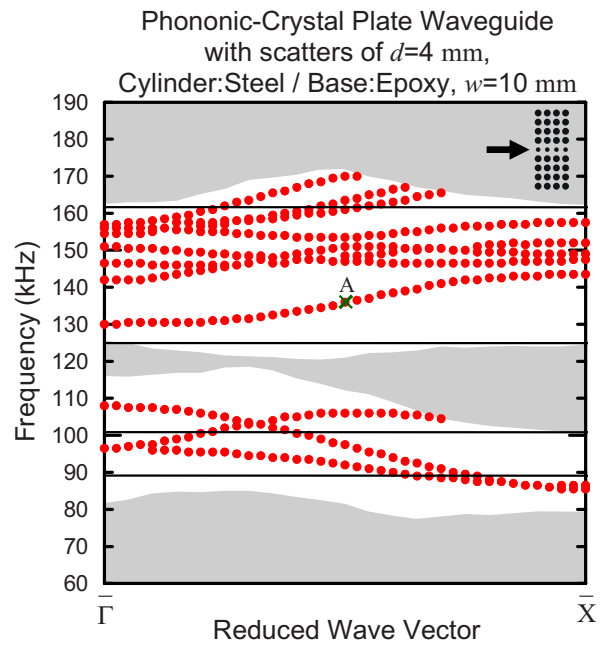

(b)

FIG. 8. (Color online) (a) Top view of a waveguide with scatters in the phononic-crystal plate and the supercell. (b) Band structure of acoustic waves in the steel/epoxy phononic-crystal plate waveguide of width $w=10 \mathrm{~mm}$ with the scatters of diameter $d=4 \mathrm{~mm}$.

guide have a smaller diameter of $4 \mathrm{~mm}$. This means that one row of cylinders in the phononic-crystal plate is replaced by the smaller ones in radius. Thus, the waveguide is consisted of multiply unit cells. After defining the corresponding supercell, the band structure of the waveguides is calculated and presented in Fig. 8(b). In the dispersion diagram, there are 11 bands of defect modes in the complete band gaps. However, there is a single-mode band range from 130 to $142 \mathrm{kHz}$ at the second complete band gaps. In addition, there is a forbidden gap at $125-130 \mathrm{kHz}$. We demonstrated the propagation of the $136 \mathrm{kHz}$ acoustic wave in Fig. 9(a). From the corresponding mode marked as point $A$ in the dispersion curve, the wave vector $\mathbf{k}$ is $(0.5 \pi / a, 0)$, and thus the wavelength is $2 a$. The amplitude field of the wave is affected by the scatters, but the periodic pattern satisfies the foregoing calculation. The characteristic displacement field of this mode is shown in Fig. 9(b). In addition to the 3D vector plot, two $2 \mathrm{D}$ figures of the cross section at the center of waveguide and a half lattice constant away from the center are also plotted. The sizes of cones in these 2D figures are normalized independently. From these displacement patterns, the wave is also identified as a flexural mode and the polarization varies along the $x_{2}$ direction. Since the polarization 


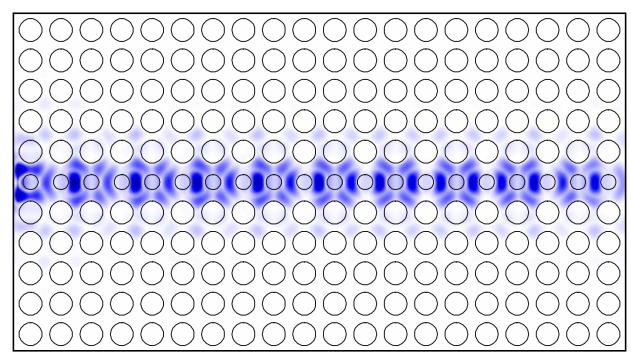

(a)
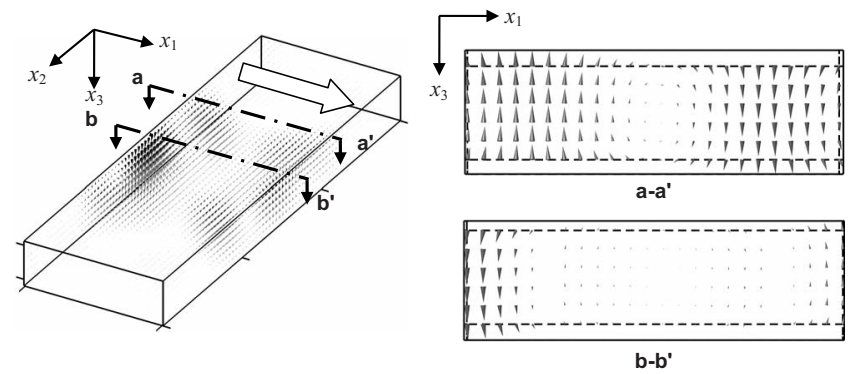

(b)

FIG. 9. (Color online) (a) Amplitude distribution of the $136 \mathrm{kHz}$ acoustic wave [point $A$ in Fig. 8(b)] on the surface of the linear phononic-crystal plate waveguide with scatters. (b) The displacement fields of the eigenmode point $A$ in Fig. 8(b).

shows symmetry with respect to the central plane (the $x_{1}-x_{3}$ plane) of the waveguide, the mode is excited by the line source of $x_{3}$ polarization. Indeed, the single-mode frequency range is useful for further applications. For example, an acoustic wave coupler can be designed based on this property.

Finally, the phononic-crystal plate of the inserted scatters, the lattice constant $a=8 \mathrm{~mm}$, thickness $h=2 \mathrm{~mm}$, and cylinders of $4 \mathrm{~mm}$ in diameter, has also been analyzed. The dispersion relation shows that there is only one complete band gap, the first one, at $101-112 \mathrm{kHz}$. The second complete band gap observed in Fig. 2 vanishes because other high frequency modes move downward. Obviously, the single row scatters do not act as the perfect phononic crystal. The band gaps and extended modes are still dominated by the original phononic-crystal plate but the scatters change the defect modes inside the waveguides.

\section{CONCLUSION}

In this paper, we have applied the FDTD method to analyze acoustic wave propagation in phononic-crystal plates and waveguides. Combining the free surface condition and $\mathrm{PBC}$, the band structure of the 2D phononic-crystal plate is calculated and the steel/epoxy phononic-crystal plate shows two complete band gaps. By investigating the displacement fields of eigenmodes, the modes similar to the one of a classical plate and some different modes resulted from the periodic structure are identified. Furthermore, waveguides based on the complete band gaps of a phononiccrystal plate are constructed. Inside the phononic-crystal plate, the waveguides have numerous defect modes that acoustic waves can propagate in. To modulate the defect modes of waveguides, the waveguides with narrowed width were calculated and the modes were depressed by the constraint. Besides, the defect modes are also changed by introducing scatters inside the waveguides. Finally, the phononiccrystal plate has a limit size in thickness which is practicable to be manufactured, and the acoustic wave is confined with the structure without the possibility of propagating away. Thus, it has potential to realize acoustic circuits on the phononic-crystal plates.

\section{ACKNOWLEDGMENT}

The authors acknowledge the financial support of this research by the National Science Council of ROC (NSC 962221-E-002-206-MY3).
*Corresponding author. wutt@ndt.iam.ntu.edu.tw

${ }^{1}$ M. S. Kushwaha, P. Halevi, L. Dobrzynski, and B. DjafariRouhani, Phys. Rev. Lett. 71, 2022 (1993).

${ }^{2}$ M. S. Kushwaha, P. Halevi, G. Martinez, L. Dobrzynski, and B. Djafari-Rouhani, Phys. Rev. B 49, 2313 (1994).

${ }^{3}$ M. S. Kushwaha and P. Halevi, Appl. Phys. Lett. 64, 1085 (1994).

${ }^{4}$ Y. Tanaka and S. I. Tamura, Phys. Rev. B 58, 7958 (1998).

${ }^{5}$ Y. Tanaka and S. I. Tamura, Phys. Rev. B 60, 13294 (1999).

${ }^{6}$ T.-T. Wu, Z.-G. Huang, and S. Lin, Phys. Rev. B 69, 094301 (2004).

${ }^{7}$ T.-T. Wu, Z.-C. Hsu, and Z.-G. Huang, Phys. Rev. B 71, 064303 (2005).

${ }^{8}$ V. Laude, M. Wilm, S. Benchabane, and A. Khelif, Phys. Rev. E 71, 036607 (2005).

${ }^{9}$ J.-H. Sun and T.-T. Wu, Phys. Rev. B 71, 174303 (2005).

${ }^{10}$ M. Kafesaki, M. M. Sigalas, and N. García, Phys. Rev. Lett. 85, 4044 (2000).
${ }^{11}$ A. Khelif, B. Djafari-Rouhani, J. O. Vasseur, and P. A. Deymier, Phys. Rev. B 68, 024302 (2003).

${ }^{12}$ A. Khelif, B. Djafari-Rouhani, J. O. Vasseur, P. A. Deymier, Ph. Lambin, and L. Dobrzynski, Phys. Rev. B 65, 174308 (2002).

${ }^{13}$ Y. Pennec, B. Djafari-Rouhani, J. O. Vasseur, A. Khelif, and P. A. Deymier, Phys. Rev. E 69, 046608 (2004).

${ }^{14}$ Y. Tanaka, T. Yano, and S. I. Tamura, Wave Motion 44, 501 (2007).

${ }^{15}$ J.-H. Sun and T.-T. Wu, Phys. Rev. B 74, 174305 (2006).

${ }^{16}$ J.-C. Hsu and T.-T. Wu, Phys. Rev. B 74, 144303 (2006).

${ }^{17}$ A. Khelif, B. Aoubiza, S. Mohammadi, A. Adibi, and V. Laude, Phys. Rev. E 74, 046610 (2006).

${ }^{18}$ Y. Tanaka, Y. Tomoyasu, and S. I. Tamura, Phys. Rev. B 62, 7387 (2000).

${ }^{19}$ P.-F. Hsieh, T.-T. Wu, and J.-H. Sun, IEEE Trans. Ultrason. Ferroelectr. Freq. Control 53, 148 (2006).

${ }^{20}$ I. E. Psarobas, N. Stefanou, and A. Modinos, Phys. Rev. B 62, 5536 (2000). 